\title{
EVALUATION OF FAT AND ITS CONTENTS OF FATTY ACIDS IN SAMPLES OF HEN EGGS MARKETED IN RIYADH CITY-SAUDI ARABIA
}

( Received: 30 . 7. 2007)

\author{
By \\ N. A. Al-Badr \\ College of Food Sciences and Agriculture, King Saud University, Riyadh, Saudi Arabia
}

\begin{abstract}
Hen egg is an easily digestable nutritious food item. It is considered a reference protein for other food proteins. Egg yolk is a rich source of lipids as it contains about 30-33\%of lipids. In recent years the lipid composition of chicken egg has been an area of primary consumers and researchers concern, due to the relation between omega 3 and omega 6 fatty acids .Many studies recommended fortification of laying hens diet with sources of these fatty acids.

The objective of this study was to evaluate the fatty acid composition and the fat content of some types of hen eggs marketed in Riyadh city. Four types of eggs were collected from the local supermarkets. Egg weight and components as well as egg yolk fatty acid composition were evaluated. Also fat and cholesterol contents were recorded.

The results of this study revealed that egg weight ranged between 51.82 to $63.05 \mathrm{~g}$., percentages of yolk and white ranged between 26.81-33.79 and 51.86-63.12, respectively.

As for the fat and cholesterol contents, they were less than $30 \%$ and above $20 \%$, respectively. The fatty acid composition of the egg yolk showed that the total saturated fatty acids was more than $40 \%$ with palmitic acid as the dominant one. Total polyunsaturated fatty acids were less than $20 \%$. Oleic acid was the major monounsaturated fatty acid. Low omega 3 and omega 6 values were observed for all samples and might be due to their percentages in the diet.

Due to health benefits of these fatty acids (omega 3and 6) the study recommended the fortification of hen diet with sources of these fatty acids.
\end{abstract}

Key words: chicken egg,egg yolk, fat, fatty acids.

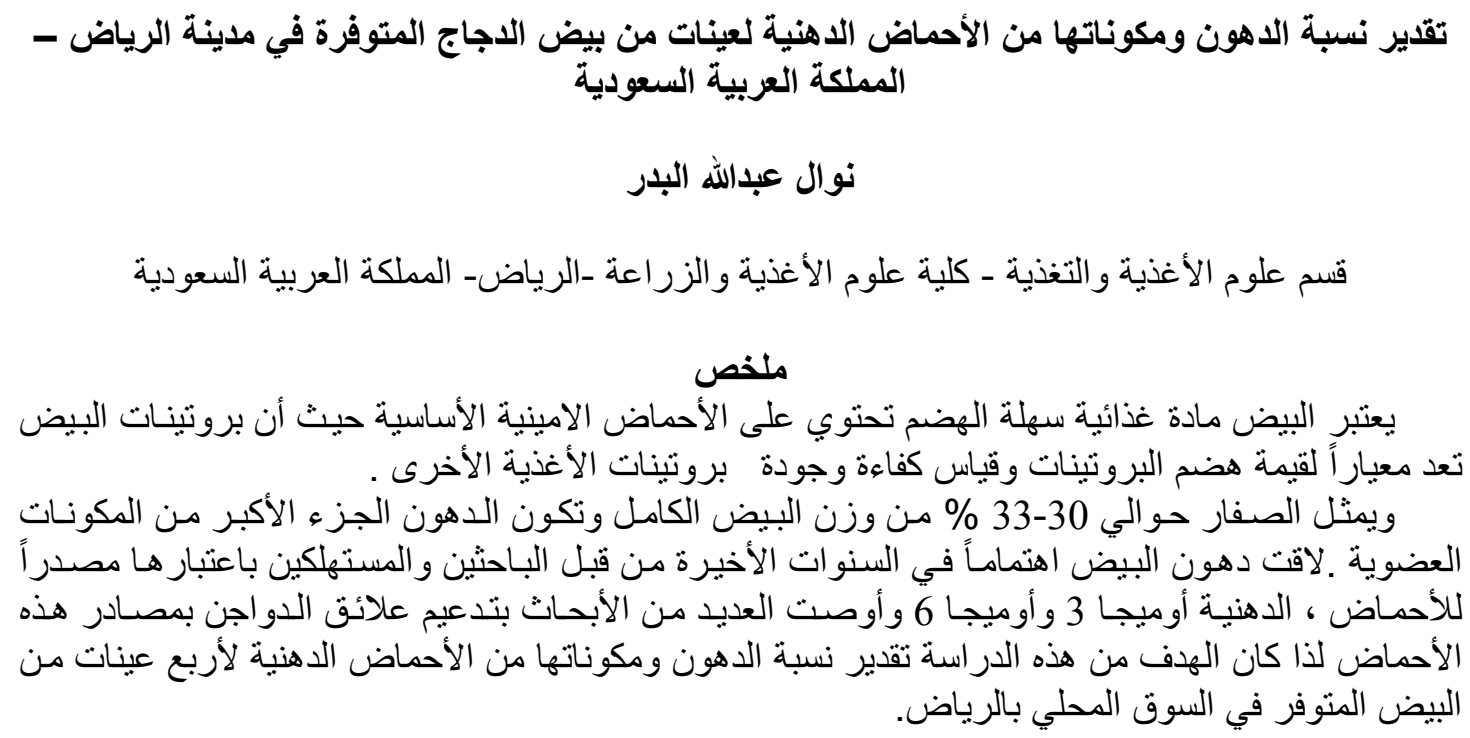


أوضحت نتائج هذه الدراسة أن وزن البيضة الكاملة تر اوح ما بين 51,82 جر ام إلى إلى 05, 63 جم أما نسبة

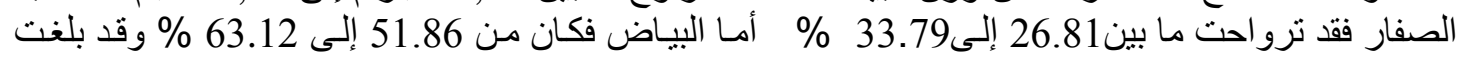

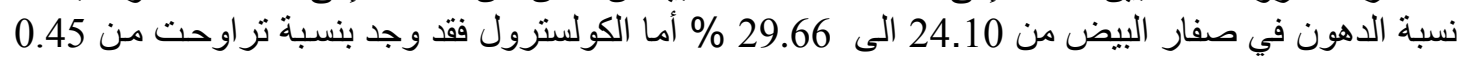

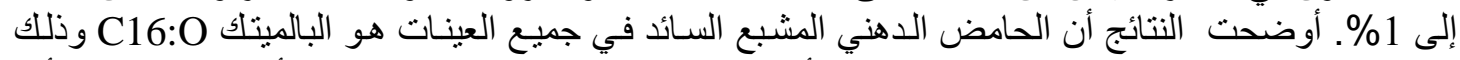

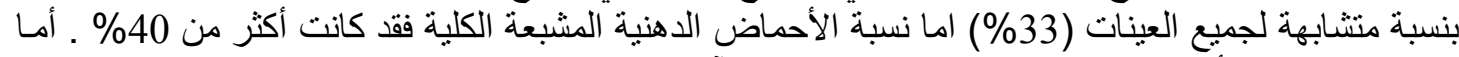

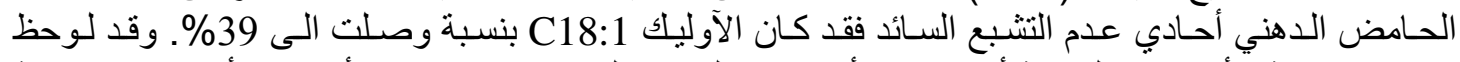

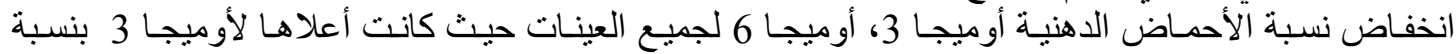

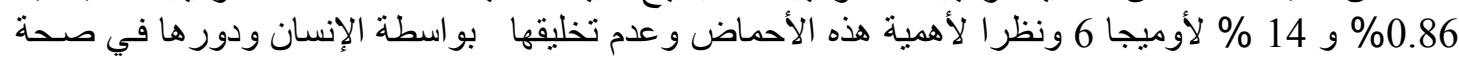
الفرد توصي الدر اسة بتدعيم علائق الدجاج البياض بمده الاصدادر هذه الأحماض.

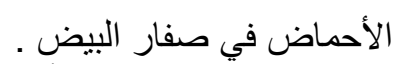

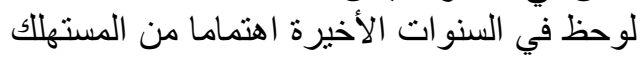

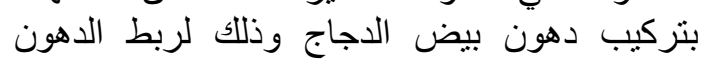

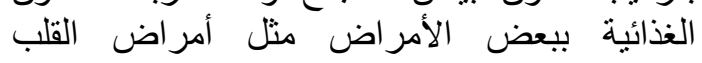
و السرطان (Simopoulos and Salem,1992)

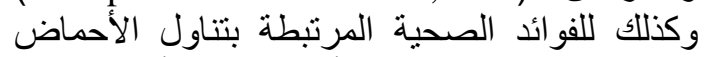

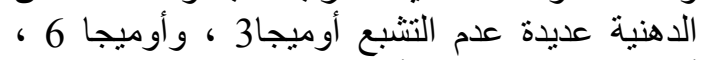

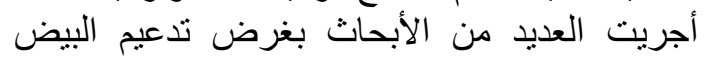

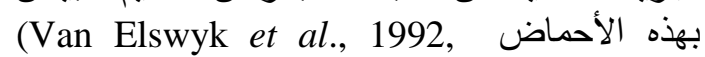
ومن المعروف ان Harris, 2003) 20:4 omega, 6 18:2omega6 أساسيان في اوميجا6 و اللينولينك

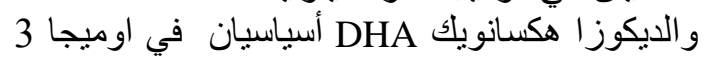
يمكن ان يخلق الارشدونيك من اللينوليك بينما في اليكان اليكان

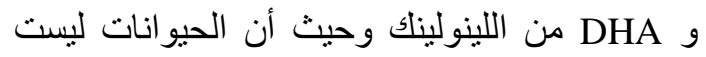

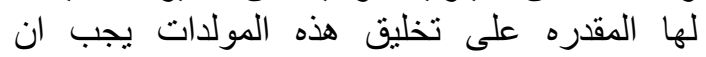

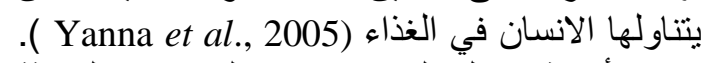
ترجع أهمية تناول البيض حسب الدار النات الدات الحديثة

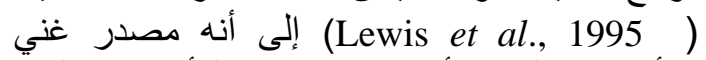

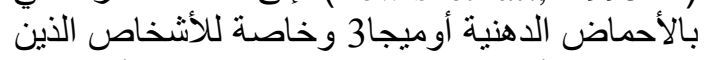

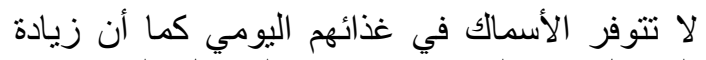

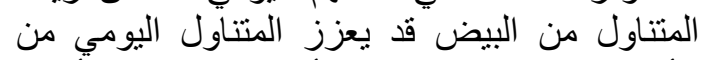

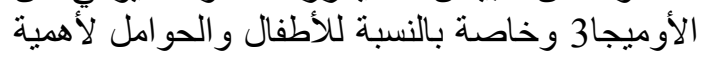

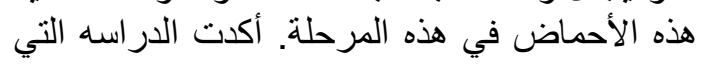

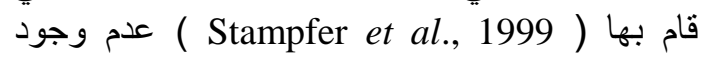
علاقة بين استهلاك البيض بكمية تصل إلى بيض وإنه

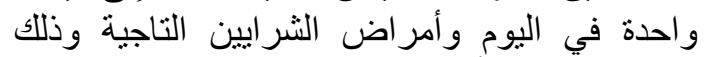

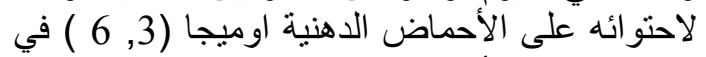

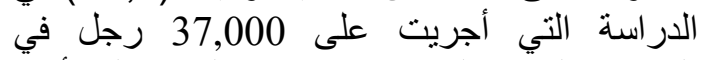

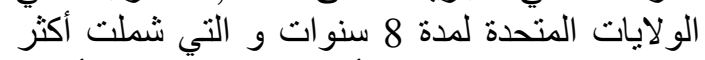

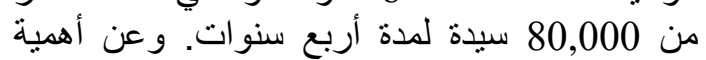
البيض كمصدر للأحماض الدهنية أوميجا 3 أشار ألى ألى (Cunnane et al., 2000)

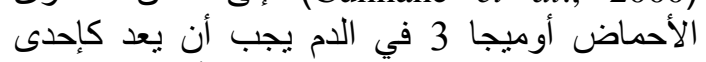

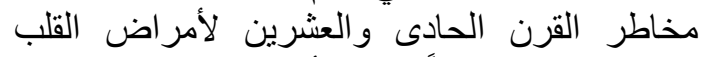

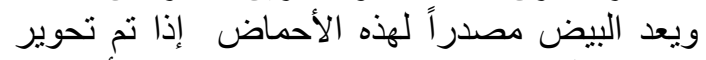

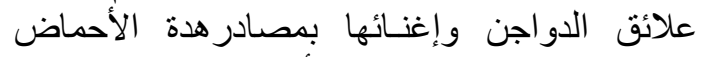
(Marcele et al., 2006)

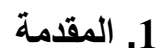

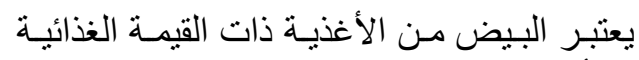

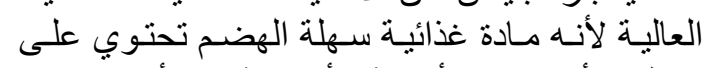

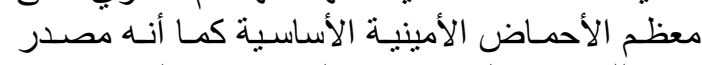

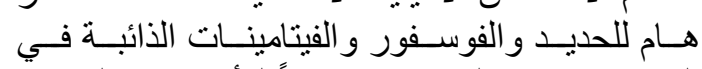

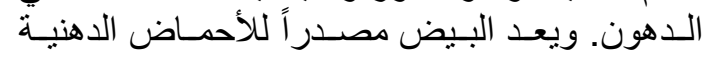

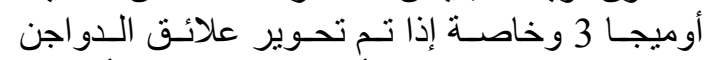

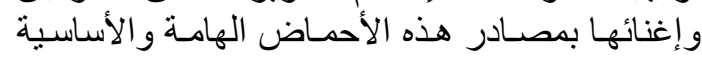

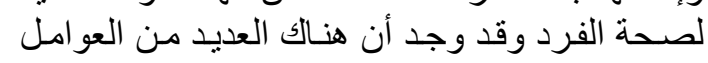

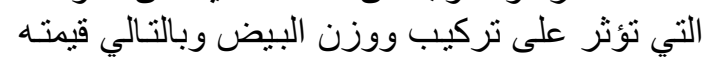

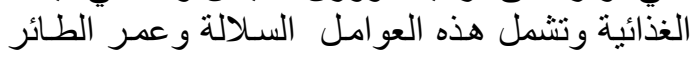

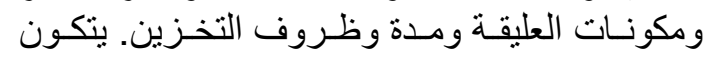

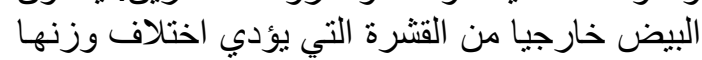

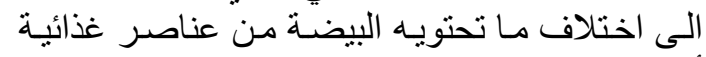

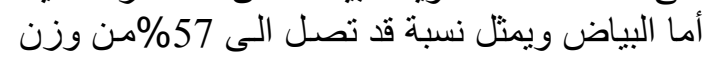

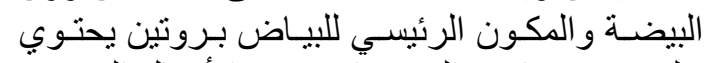

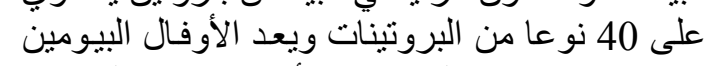
Ovalbumin

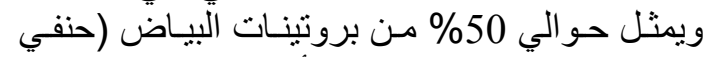

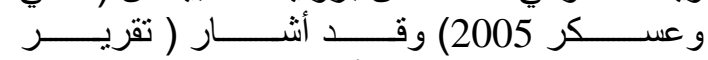

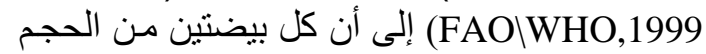

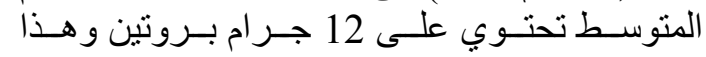

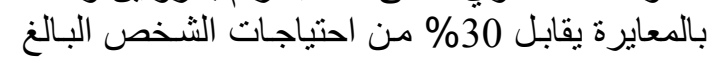

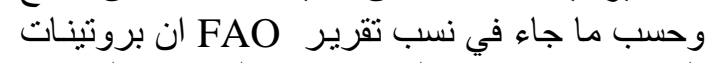

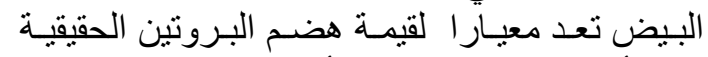

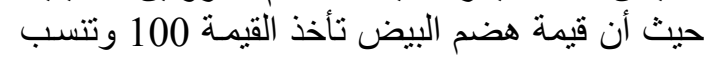

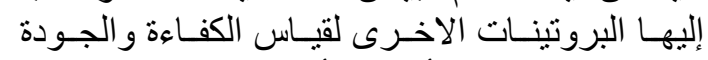

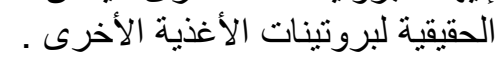

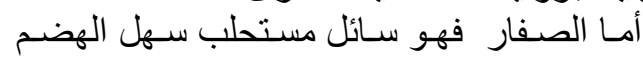

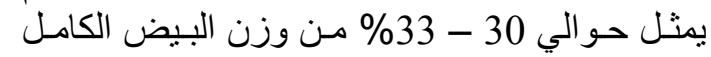

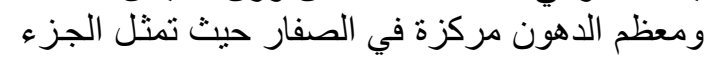

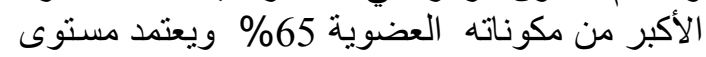
الدهون ومكوناتها على العليقة وقد أنشار

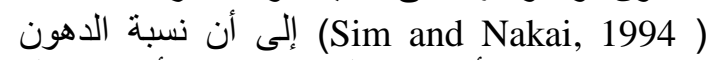

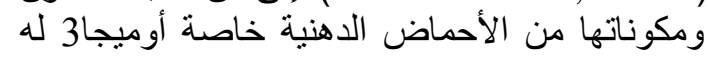

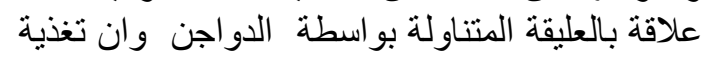

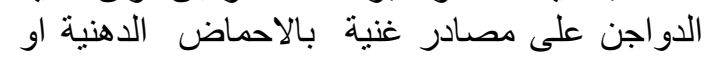
ميجا من شانه ان يرفع مستوى هذه بالاحن 
الأحمـاض الدهنيـة القياسية للتعرف على الأحمـاض

الدهنية المفصولة وفقا لطريقة (AOAC,1995 ) جـ - تقدير الكولسترول :

تم تقدير نسبة الكلسترول فى صفار البيض لكل

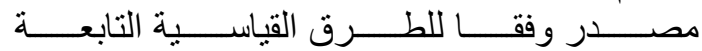

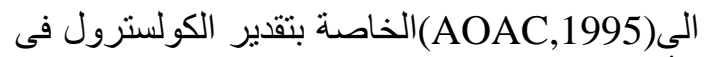

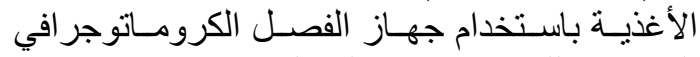
الغازي وذللك بعد إتمام عملية التصبن.

\section{3. النتائج والمناقشة}

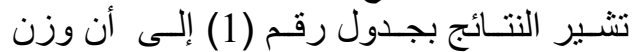

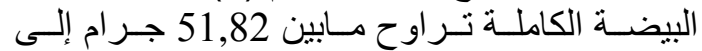

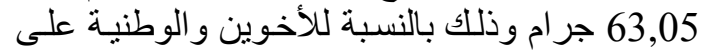

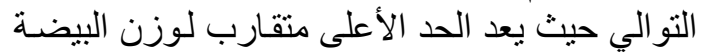

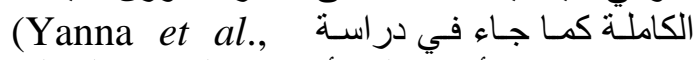

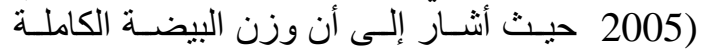

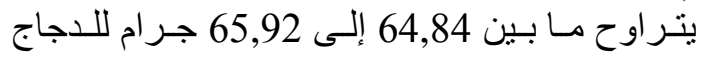
الـذي يتنـاول العليقـة المرجعيــة المعروفـة للــدجاج
الكلسترول و هو مكون طبيعي في صفار البيض فقط

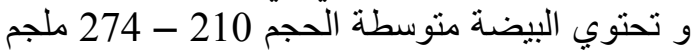

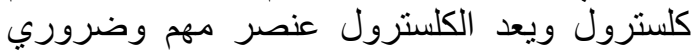

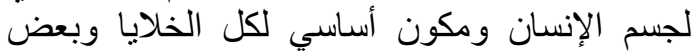

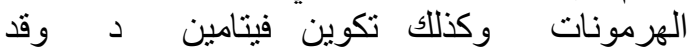
Sin and Nakai,1994a,\&b) Siscovick (1997) اشنسار ,) إلى أن استهلاك البيض بطريقة معتدلة لا تؤدي إلى رفع مستوى الكلسترول وذللك لكمية تصل الكل الى لى

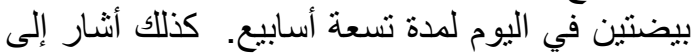

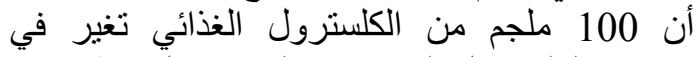
مستوى الكلسترول الى 20,5 ملجم / دسل فقط .

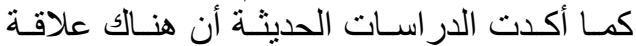

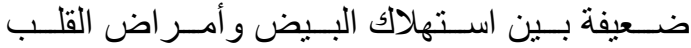

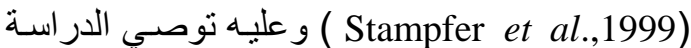

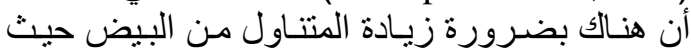

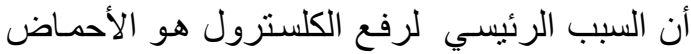

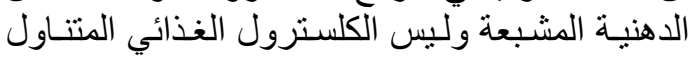
في أغذية مثل البيض .

\begin{tabular}{|c|c|c|c|c|c|c|}
\hline & & & & \multicolumn{3}{|c|}{ جدول (1) النسب المئوية لمكونات البيض. } \\
\hline وزن & 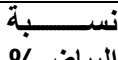 & وزن البياض & نسبة & وزن & وزن البيضة & أجزاء البيضة \\
\hline جم & 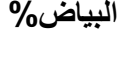 & جم & 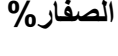 & الصفار & (الكاملة & \\
\hline 7,91 & 56,64 & 32,15 & 29,42 & 16,70 & 56,757 & 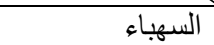 \\
\hline 7,81 & 56,55 & 33,65 & 30,32 & 18,04 & 59,496 & فقيه \\
\hline 9,04 & 51.86 & 32,70 & 33.79 & 21,31 & 63,053 & 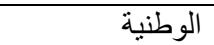 \\
\hline 5,21 & 63,12 & 32,71 & 26,81 & 13,90 & 51,824 & الأخوين \\
\hline
\end{tabular}

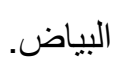

أمسا الصفار فقد نراوحت نسبتة مـابين إلى 26,81 وذلك بالنسبة للوطنيـة و الأخـوين على فئى التوالي وتعتبر نسبة الصفار في عينات البيض تلحت

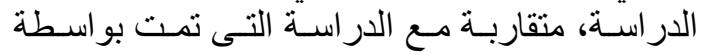
التى أشارت إلى أن (Sim and Nakai, 1994 a\&b)

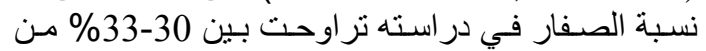
وزن البيضه الكاملة. أما نسبة البياض فقد كانت متقاربة بالنسبة للأخوين

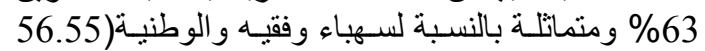
\%) وذلك بالر غم من التفاوت في وزن البيضة الكاملة .

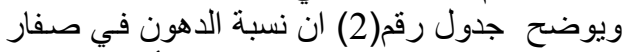

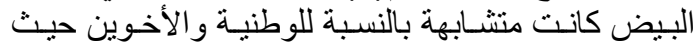

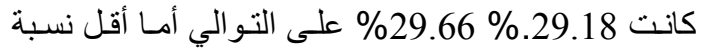

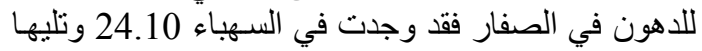
فقيه 28.91 ويعتمد تركيز الدهن في الصفار فئه إلى حد كبير

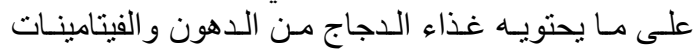

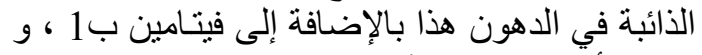

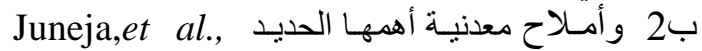
(1997). أمـا عن نسبة الكلسترول في الصـفار فقد

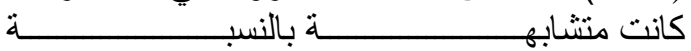

ونظر الأهمية البيض الغذائية ودوره في صحة

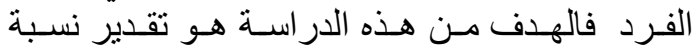

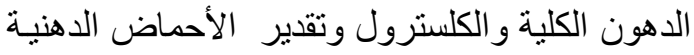

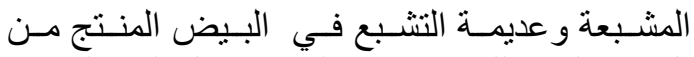
الدجاج المستهلك في مدينة الرياض بالمملكة العربيـة

\section{2.المواد والطرق المستخدمة في البحث}

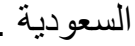

تم اختيار بيض الاجاج من السوق المحلي بمدينة

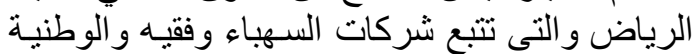
والاخوين وتمثل الاكثر استهلاكا. 0202 الطرق المستخدمة للتقدير الاكثر التخاير أـ تقدير نسبة الدهن في صفار البيض البن : تم فصل

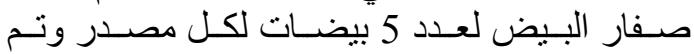
تجنيسـه ثم استخلاص وتقدير الدهن الكلي بواسطة

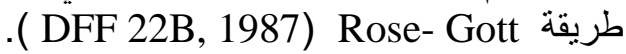
ب ـ تقدير الأحماض الدهنية :

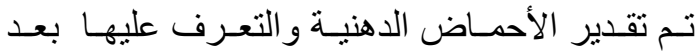

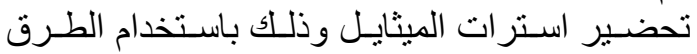
الكروماتوجر افية للفصل - وتم استخدام خليط مـن بلـن 
حامض الأوليك في عدم رفع الكلسترول لأنسه المـادة

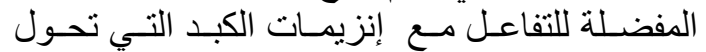
الكلسترول إلى الصـورة غير النشطة ( , . Grundy

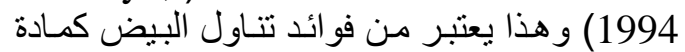

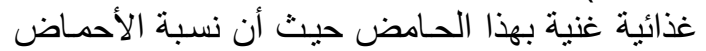

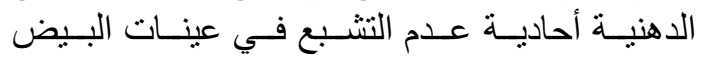

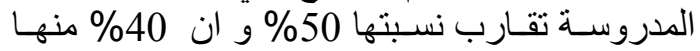

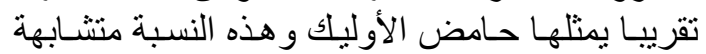

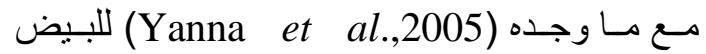

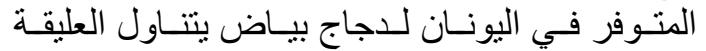

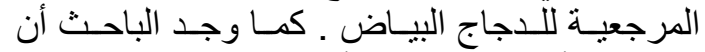
مجموع الأحماض الدهنية أحاديـة عدم التشبع كانت

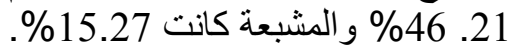

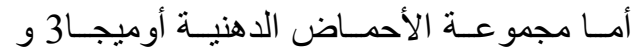

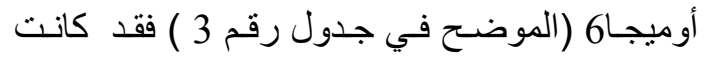

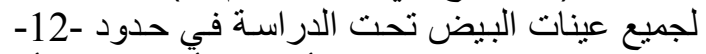

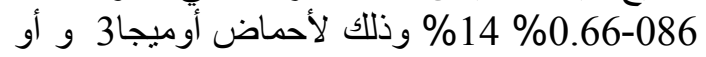

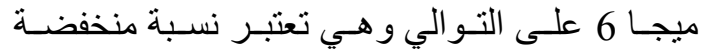

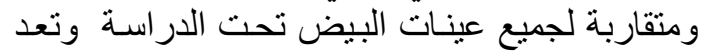
منخفضة مقارنة بالعينات المغذاة على عليقة مرجعية

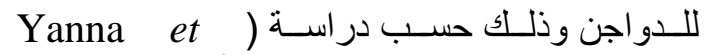
(al.,2005 حيث كانت مجموعة الأحماض الدهنية

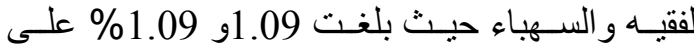
التو الي و انخفضت النسبة لكل من الوطنية والأخوين حيث كانت 0.88، 0.45\% على التوالي.

جدول رقم (2) نسبة الدهون الكلية و الكلسترول

\begin{tabular}{|c|c|c|}
\hline الكولسترول\% & الكلية\% الدهون & مصدر البيض \\
\hline 1,02 & 24,10 & السهباء \\
\hline 1,09 & 28,91 & فقيه \\
\hline 0,88 & 29,66 & الوطنية \\
\hline 0,45 & 29,18 & الأخوين \\
\hline
\end{tabular}

وبالنسبة لمحتوى عينـات البيض من المصـادر

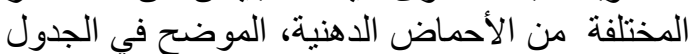
رقم (3)، وجد أن الحامض الدهن الدني المشبع الَّائد في

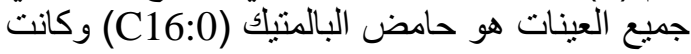

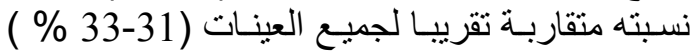

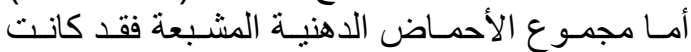

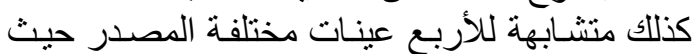

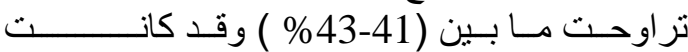

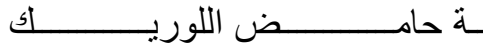

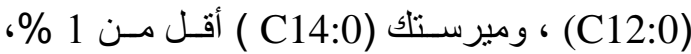

جدول (3) الأحماض الدهنية في عينات البيض المختلفة تحت الدراسة .

\begin{tabular}{|c|c|c|c|c|}
\hline \multicolumn{4}{|c|}{ عينات البيض من المصادر المختلفة } & \multirow[t]{2}{*}{ الأحمـاض الدهنية } \\
\hline 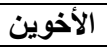 & الوطنية & فقيه & السهباء & \\
\hline 30.83 & 33.70 & 33.00 & 33.34 & Palmitic acid C16:0 \\
\hline 9.41 & 9.48 & 9.56 & 8.84 & Stearic acid C18:0 \\
\hline 39.21 & 37.57 & 36.86 & 38.10 & Oleic acid C 18:1 \\
\hline 12.74 & 11.62 & 13.59 & 11.44 & Linoleic acid C 18:2 \\
\hline 1.26 & 1.22 & 1.11 & 1.14 & Linolenic acid C 18:3 \\
\hline 1.26 & 1.22 & 1.11 & 1.14 & Archidonic acid C20:4 \\
\hline 0.21 & 0.31 & 0.31 & 0.38 & Eicosapentaenoic acid C20:5 \\
\hline 0.27 & 0.21 & 0.20 & 0.20 & Docosahexanoic acid C22:6 \\
\hline 40.24 & 43.18 & 42.56 & 42.18 & Total saturated fatty acids \\
\hline 39.21 & 37.57 & 36.86 & 38.10 & Total mono un saturated fatty acids \\
\hline 14.67 & 13.50 & 15.41 & 13.44 & Total poly un saturated fatty acids \\
\hline 0.67 & 0.66 & 0.71 & 0.86 & Total Omega 3 fatty acids \\
\hline 14.00 & 12.84 & 14.70 & 12.58 & Total Omega 6 fatty acids \\
\hline
\end{tabular}

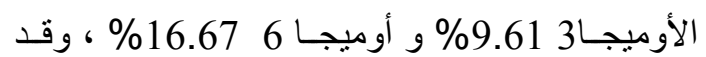
يرجع سبب الانخفاض في الأحماض الدهنيـة أوميجا

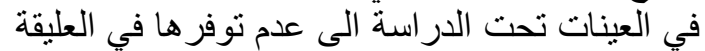

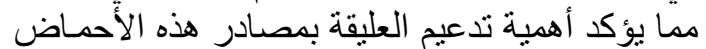

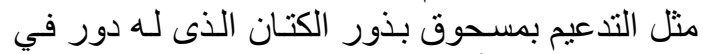

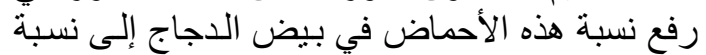

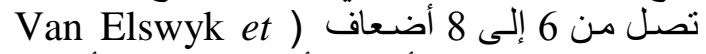
3al.,1992). ونظر الأهمية الأحماض الدهنية أوميجا

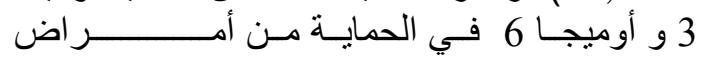

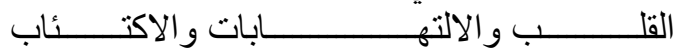

لجميع العينات. ومن المعروف أن هذه الأحماض لها

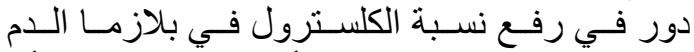
كمـا أنشـار الباحـث إلى أن (Grundy, 1994)

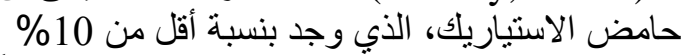

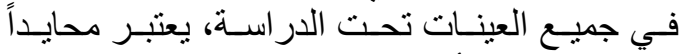

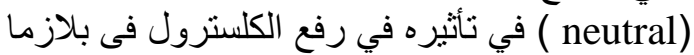
الدم. كما يوضح الجدول رقم (3) اليره في أن حامض الأوليك

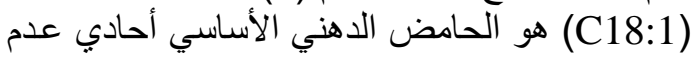

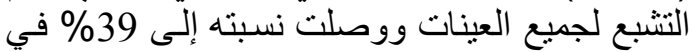

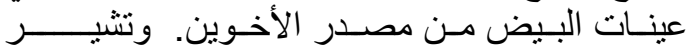

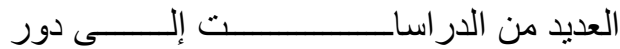




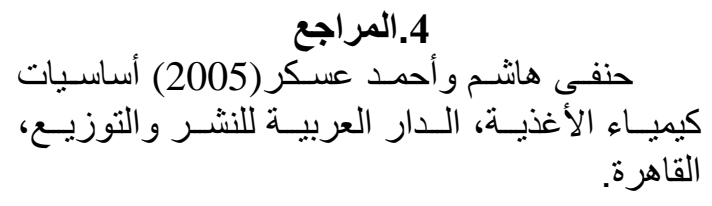

AOAC. (1995). Official Methods of Analysis of AOAC International $16^{\text {th }}$ ed. Arlington Virginia U.S.A.

Cunnane S.C.,Francescutti,V.,Brenua,J.T., and Rawford,M.A..(2000).Breast-fed infants achieve a higher rate of brain and whole body DHA. accumulation than formula-fed infants not consuming dietary DHA.Lipids, vol 35(1): 105-111.

D F F22B,.(1987). Skimmed milk, whey and butter milk determination of fat content Rose Gottilieb gravimetric method.

FAO/WHO (1999). Protein quality evaluation report of the joint FAO/WHO Expert consultation on protein quality evaluation. Held in Bethesda, MD.Dec. 4-8,1989. Food and Agriculture Dramatization of the United Nations, Rome, Italy.

Grundy S.M., (1994). Influence of stearic acid on cholesterol metabolism relative to other long-chain fatty acids Am.J.Clin.Nutr.vol60(1):.986-90.

Hardman W.E,.(2002). Omega-3 fatty acids to augment cancer therapy. J. Nutr. Vol132(1): . 3508s-3512S).

Harris,W.S,(2003)n-3Long-chain polyunsaturated fatty acids reduce risk of coronary heart disease death; extending the evidence to the elderly. Amer.J.of Clinical Nutri. Vol. 77(2):.,279-280.

Juneja,L.R,. Yamanoto, L.R.H.Juneje, M.Hattg. and.Kim, M. (1997). Egg yolk lipids hen eggs :.973-980.

Lewis N.M.,Widga AC.Buck.J.S and .Frederick A.M.(1995).Survey of omega 3fatty acids in the diet of Mid west Low-income pregnant women. J. Agromed Vol. 2: 49-57.
وكذلك أهميتها للمرضع و الاطفال وخاصة في مرحلة

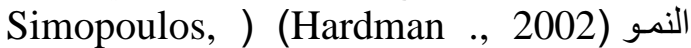

2002) لذا توصـي الدراسـة بتدعيم عليقة الدو اجن

البياض بمصادر أوميجا 3 و 6 التئة

Marcele S., Edward B. and John D,.(2006) Human Health Effects of fatty acids in Beef Extension Services West Virginia University.

Simopoulos , A.P.and Salem N.(1992). Egg yolk as a source of long-chain polyunsaturated fatty acids in infant feeding. Am,.J.Clin.Nutri., vol 55: 411-414.

Simopoulos A.P. (2002). The importance of the ratio of omega-6lomega-3 essential fatty acids. Biomed Pharmacother, Vol. 56(8) : 365-79

Sim,J.,and Nakai, S,.(1994a). Egg uses and processing technologies. Oxon: $\mathrm{CAB}$ International.

Sim, J. and Nakai, S. (1994b). Whole body and hepatic cholesterol synthesis rates in serum lipids and effect of dietary olive oil quality. J. Lip. 31,11491172.S.

Siscovick D S.(1997)..Exercise and its role in sudden cardiac death. Cardiol Clin.Nutr.,15:467-72.

Stampfer Hu FB. MJ.Rimm EB., Manson J.E.,Ascherio,A.Colditz,G.A.,Rosner B.A.,Rosner,B.A.,Speigelm,D.,Speize r,F.E., Socs., F.R.,Hennekens, C.H., and Wilett W.C(1999). A prospective study of egg consumption and risk of cardiovascular disease in men and women. JAMA Vol. 281:pp.1387-94

Van Elswyk. M.E., Sams, A. R. and Hargis P.S.(1992).Composition, functionality and sensory evaluation of eggs from hens fed dietary menhaden oil. J. Food Sci., Vol. 57:.342-349.

Yanna K.A.,Teserveni. G,. and Christaki, E.(2005).Enhanced egg production in practice: The case of Bio-Omega-3 Egg. Hnter.J. of Poultry Sci., Vol. $4(8)$ :p.531-535. 